\title{
Mechanisms Lead towards Improved Health: Massage with Earthing
}

\author{
Madiha Khalid and Jeremy Madvin
}

\section{ABSTRACT}

\begin{abstract}
Massage can alleviate the inflammatory process, help to accelerate recovery, and relieve pain resulting from muscular injuries. In this paper, we combine the idea of mechanotransduction (conversion of mechanical stimulus into electrochemical activity) using grounding in massage to investigate beneficial mechanisms. Grounding massage has been discovered to stabilize the physiology of the body, help reduce pain, inflammation, stress, enhance sleep, increase energy, and blood flow, and enhance wellbeing by connecting the body with the electrical charges of the Earth. Such effects are systemic and basic, and often quickly evolve. This process can reduce secondary injury, collateral sprouting, and nerve sensitization by modifying inflammatory signaling pathways, resulting in improved damage recovery and pain reduction or prevention. Research shows that grounding, when simultaneously combined with many CAM clinical practices, can offer great potential to improve their effectiveness. The paper aims to explain how massage therapy, combined with geographically and simultaneous earthing, causes a potentially beneficial and properly activated immunomodulatory pathway.
\end{abstract}

Keywords: Earthing, Electron deficiency, Grounding, Inflammation, Massage.
Submitted : April 12, 2021

Published : May 03, 2021

ISSN: 2593-8339

DOI: $10.24018 /$ ejmed.2021.3.3.812

\section{Dr. Madiha Khalid *}

Humdard University, Islamabad Campus, Pakistan.

(e-mail: msmadihakhalid@gmail.com) Jeremy Madvin

MBA International Business

The University of Redlands, United

States.

(e-mail: Jeremymadvin@gmail.com)

*Corresponding Author

\section{INTRODUCTION}

Complementary and alternative medicine (CAM) is becoming increasingly popular day by day. It encompasses various treatments, from nutritional supplements to chiropractic and massage therapies provided by practitioners [1]. People receiving CAM services incur out-of-pocket expenses, equivalent to those of a family physician in a given year. According to experts, the most commonly cited reason for seeking CAM therapy, such as massage, is treating musculoskeletal disorders or diseases resulting in pain [2]. The majority of people use complementary and alternative medicine (CAM/CIM) in addition to conventional treatments, with "symptom relief" being one of the most common reasons [1].

Massage therapy and grounding have been promoted to treat many conditions, including stress, musculoskeletal disorders, and pregnancy [3]. These therapies have become widely accepted to treat musculoskeletal disorders due to pain alleviation and functional improvement. In practice, massage and grounding treatments have proven to be one of the most primitive therapeutic tools used to alleviate pain and inflammation [2].

This paper explores how massage therapy, combined with grounding, influences inflammatory responses and control pain. This paper begins with an overview of massage and grounding so that the reader will gain a comprehensive understanding of these therapies, including how electric charges work and how endogenous chemicals are produced during the process affect pain transmission through afferent nerve fiber activation. This paper will emphasize the concept of mechano-transduction and its implications to cellsignaling pathways. Clinicians would be better able to make fully informed choices about therapies for musculoskeletal injuries if they were to have a better understanding of the physiologic effects of combining the outcomes of both grounding and massage on cellular processes underlying inflammatory pathways and pain control.

\section{A. Massage Therapy}

Massage therapy, which is characterized as manipulating soft tissue areas of the body, is used in clinical settings to help people relax, sleep better, and alleviate muscular aches and pains. The use of human touch as a treatment for pain and other physiological concerns has a strong appeal. It typically offers a non-invasive, low-cost adjunct to treating pain and other symptoms in patients with acute or chronic illnesses. [4]. Massage therapy is characterized in a variety of ways. An Ottawa panel recently described massage as "soft tissue and joint manipulation with the hands or a handheld device" [5]. The use of mechanical devices and (spinal) manipulation were also included in this category. Massage is also described as "a systematic manipulation of the soft tissue using rhythmical pressure and stroking to prevent, improve, sustain, 
rehabilitate, or improve physical function or ease pain" [6]. Popular massage techniques include effleurage (a gliding or sliding motion across the skin), friction (penetrating pressure applied through the fingertips), petrissage (lifting, wringing, or squeezing of soft tissues in a kneading motion, or pushing or rolling of the tissues), tapotement (rapidly striking the tissues), and vibration [7]. Massage therapy's exact mechanisms are unclear, but diverse physical responses to massage treatment have been recorded. Increased lymph flow, a switch from sympathetic to parasympathetic response, fibrosis prevention, increased lactate clearance from blood, and effects on the immune system, memory, and pain are among the known mechanisms [8]. Massage therapy, according to common belief, may improve blood flow to the muscles. This argument, however, has been challenged, as there has been an increase in scientific evidence which has shown that massage therapy may have little effect on blood flow. Massage appears to cause local biochemical changes, contributing to increased neural activity in the spinal cord and subcortical nuclei, influencing mood and pain perception [9]. As a result, massage therapy can reduce anxiety, depression, and pain by increasing serotonin and endorphin levels [10].

\section{B. Earthing (Grounding)}

Earthing implies connection of the human body to the regular and active electrical charge present in the Earth. It is a natural lifestyle exercise that systemically affects the body's fundamental bioelectrical function. Grounding stabilizes physiology remarkably, may decreases pain and inflammation, increases flow of the blood and venous/ lymphatic flow back to the heart, enhances sleep, and decreases anxiety and stress, creating greater well-being. There are structural consequences that appear to be profound. People also say that they look and feel younger and healthier after grounding. Many with pain experience less pain [11]. Even morale increases [12]. Grounding is simple to perform and, especially for people with chronic health problems, often produces fast results.

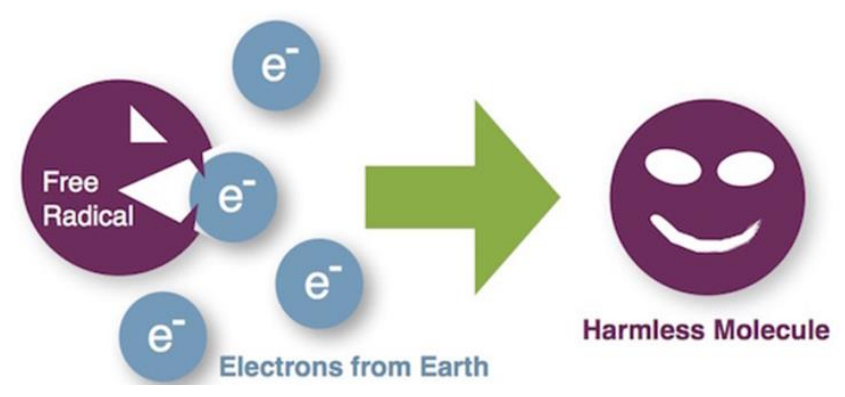

Fig. 1. How electron from Earth neutralizes the free radical.

Grounding has two basic types: 1) indoors, using economic Grounding devices (conductive sheets, mats, hand and body patches or bands) either during sleeping or working, and 2) outdoors, barefoot in contact with natural conductive surfaces such as grass, stone, dirt, sand, and gravel. It is the most common and natural form of earthing.

Due to modern human lifestyle, grounding restores a primaeval electrical link to the Planet. Grounding seems to fix an "electron deficiency syndrome," a neglected and possibly leading reason for different health circumstances [13]. Grounding is a powerful method for preventing and rehabilitating health and healing. This technique can be used independently or in combination with clinical therapies to alleviate symptoms and eliminate the root causes of diseases and disorders that start with an imbalance in the different body bioelectric levels.

It is up to the medical community to grasp that a body attached to the Earth is likely healthier than an ungrounded body. The body simply works more efficiently and under less cellular stress. Humans have been increasingly disconnected from the Planet. Obviously, now, humans typically do not sleep on the grass, do not walk outside without shoes, and almost exclusively wear insulating plastic sole shoes for more than half a century in its place of conventional and conductive leather shoes. We work and live in high-rise buildings, sometimes thousands of feet above the ground, and spend most of our time physically disconnected from the Earth.

Electrical imbalances, the buildup of harmful positively charged particles, and an unknown body deficiency of electrons can result from a lack of interaction with the Earth and cause susceptibility to malfunction, disease, and disorder. As is well known, Planet Earth's surface is electrically influenced by solar radiations, countless lightning bolts, and other dynamic forces of the atmosphere. However, it is not common knowledge that these phenomena give land masses and water bodies a continually refreshed supply of free electrons passing the Earth with a regular negative electrical charge. According to biological grounding science, this same electrical charge, with its infinite electron supply and circadian frequencies, serves as a regulating and supporting purpose for both plant and animal kingdoms, akin to "electrical nutrition." The findings back up the theory that Grounding allows many free electrons to move into the body, causing rapid physical changes, often instantaneous, as in fig 1. Grounding preserves and retains an internal electrical condition that is organically "normal". A few years ago, two Polish scholars and doctors, Sokal and Sokal, performed different experiments resulting in the conclusion that the human body is a "universal controlling force in Nature". Bioelectric, biochemical, and bioenergetic processes are highly regulated and tend to have a considerable controlling effect on chronic diseases and disorders [14].

\section{GROUNDING COMBINED WITH MASSAGE THERAPY}

Electro and chemical reactions that impact the essential biological process in the human body can be triggered by external physical stimulation. Massage therapy is also regarded in oriental medicine as induction of such reaction, allowing unstable physiological situations to return to normal physiologically balanced conditions. Massage therapy has explained the theory of physiologic massage therapy in Western medicine as a mechanism of cytological and endocrine nerve control processes [15]. The sensory system is activated by the sensory or nerve receptor neural complex in massage therapy [16]. However, while massage therapy with grounding likely results in brain responses, it may not be accurate for all reactions to occur only through the nervous system because the biological response to external stimulation through the nervous system is much faster than is believed possible. It indicates that biological methods other than the nervous system could be involved in combined 
reactions. As a result, massage therapy may be called electrical energy movement into nervous or connective tissue [17].

It has been stated that massage therapy stimulation with grounding appears to produce an ion-electric-charge, which is transmitted via various biological pathways - blocking the ion-electric-charge results in an unbalanced negative and positive charge in an organ and hence pain and the generation of multiple diseases. Grounding moves the excessive or insufficient state of the ion-electric charge to allow good health to be restored. It suggests that grounding is intricately linked to the transfer of bioelectrical charges. However, massage therapy alone does not necessarily lead to consistent, effective therapeutic outcomes. When combined with simultaneous grounding, massage therapy provides an increased likelihood of positive effect with no potential consequences.

Massage therapy has been widely used as a primary therapeutic technique due to its minimal side effects and therapeutic efficacy. However, certain conditions may affect the effectiveness of massage therapy. Although the pool of data is growing rapidly, the contribution of grounding, simultaneously combined and geographically targeted with mechanical massage therapy, has not been explicitly studied. To achieve the synergistic effect, both strategies are used together, simultaneously. This technique is generating cautious optimism of a dramatic improvement to both grounding and massage when compared to used independently.

It is known that a massager can use small e-stim tools, utilizing external power sources, to inject electrons into the human body. However, with excessive load utilizing external power sources, we can target the exact part of the body cause additional problems or harm.
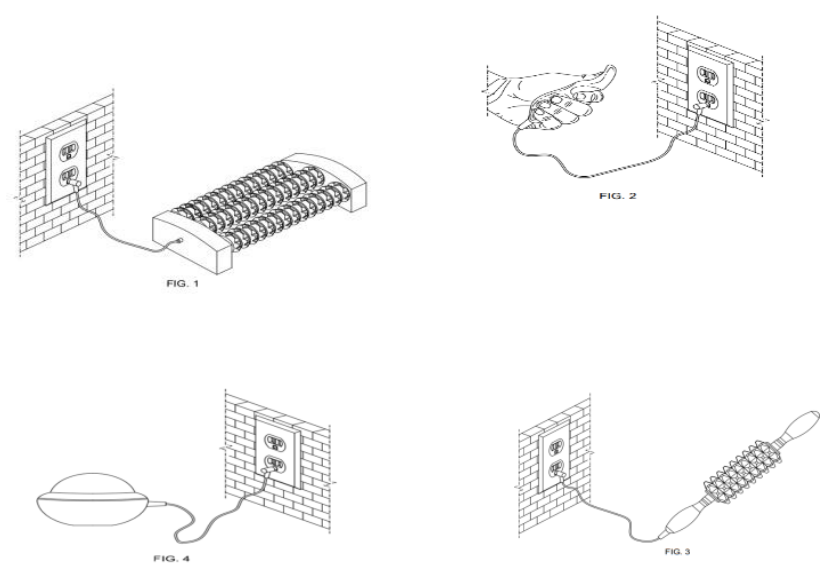

Fig. 2. Unique Earthing massagers sketches.

The above illustrations are examples of unique grounding massagers which incorporate both a grounding device and a massage device into a singular device. These devices give great flexibility to deal with specific concerns while addressing more global inflammatory or immune issues that patients have. Incorporating grounding with massage therapy using a specialized grounding massager makes it possible to precisely target the body's specific area of concern. The user can choose the precise path and exit point for the body's excess voltage and direct it through a particular part of the body or specific muscle group to increase stimulation and assist in the unblocking process.

This process of combining grounding and massage likely increases:

\section{A. Anti-inflammatory Effect}

Reducing and even removing chronic inflammation is one of the essential benefits of grounding combined simultaneously with massage. Inflammation acts as a common trigger for enhancing chronic pain and age-related diseases.

Briefly, this effect's hypothesis is as follows: free radicals are positively charged molecules that cause damage to the healthy tissue by dragging the electron from them. In normal biochemistry, free radicals are formed, including the ATP (adenosine triphosphate) production, the energy supplier of the body. Everyday billions of free radicals are generated within a human body. Grounding allows large amounts of ions to reach the body, and free radicals are assumed to be at a minimum, reduced or partially neutralized. Nervous, meridian, and circulatory systems may be involved in electron transportation's active mechanisms to an inflammation site. The inflow of ions into the human body is thought to act as potent anti-inflammatory support for the immune system. Grounding has been demonstrated to reduce inflammation quickly [18].

Grounding appears to offer the potential of a significant effect on inflammation and pain. It is clearly shown with medical thermal imaging [19] that record small changes in temperature of skin to create a colored image. See in Fig. 3.

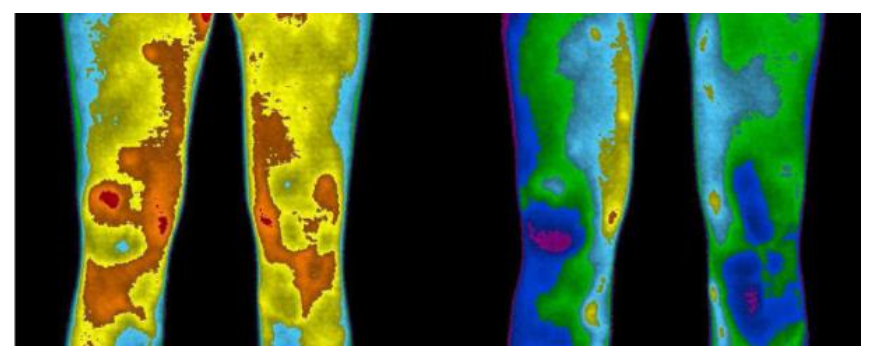

Fig. 3. Patient with knee pain. Left-half hour before grounding. Right- half hour after grounding. Left show rapid result.

\section{B. Improve Blood Flow}

Better blood flow is another important recorded advantage of grounding combined with a massage. The negative charge intensity on the red blood cell surface is called zeta potential, a factor that preserves the space between of the bloodstream cells. When the charge is higher, the cell's ability to push each other (less clumping) becomes greater, blood viscosity reduces, and the result is stronger flow. An average bloodthinning effect is indicated by the increase measured in this analysis. The referenced scientists concluded that reduced blood clumping and reduced viscosity when grounding is combined with massage appears to be one of the easiest but most in-depth steps to help reduce risk of cardiovascular incidents [20].

An additional benefit for users is that it is possible to complete these procedures quicker, getting them in and out of the clinic. Studies have shown that after about 25 minutes, the benefits of electro-acupressure are maximized. 
Chevalier got imaging proof for the first time in 2014, using a Speckle Contrast Laser camera, showing that Grounding improves blood flow to face and improves control of Autonomic nervus system on peripheral circulation. The research discovered exciting and distinct pattern in the blood flow of the face of grounding subjects as compared to nongrounded subjects [21]. This pattern correlates to the ANS's more effective regulation of blood flow and explains how grounding enhances repair and recovery of facial skin and tissues, thus generating a conventionally desired beauty impact.

Various therapies used in the cosmetics industry aim to improve facial microcirculation. Fig. 4 depicts the imaging findings of one of the study's 40 participants.

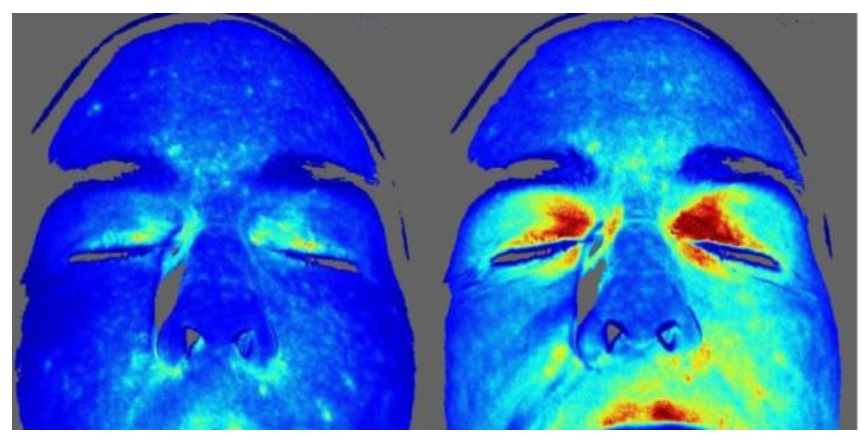

Fig. 4. After 20 mint of earthing, facial circulation improved (right image) (dark red $=$ highest flow, dark blue $=$ lowest flow $)$.

Other mechanisms associated with earthing, according to Chevalier, include increased fluidity of blood (zeta potential), normalized levels of the stress hormone cortisol which produces a relaxing effect on the body, decreased inflammation - and enhanced overall physiology of the body. Stress reduction and mood enhancement are additional significant advantages of grounding, which can be achieved by a stabilizing effect on the autonomic nervous system and cortisol control, a stress hormone [22].

Blood pressure is likely to be affected by a combination of the effects discussed in this article. Different case studies are available, showing a marked improvement in people's blood pressure after they begin grounding on a regular basis [23]. All 10 hypertensive patients who took part in the first-ever clinical trial to look at this relationship saw improvements in their blood pressure within a few months of beginning regularly grounding, and in several cases, participants were noticeably better balanced within a limited time.

\section{DISCUSSION}

Massage therapy is currently linked to various indications and contraindications, with limited research-based evidence to back up common and current recommendations. Owing to the lack of randomized control trials, many CAM therapy critics ignore its proposed results.

To demonstrate the beneficial outcomes resulting from massage therapy, numerous experiments have been carried out. However, most research has concentrated on examining massage efficacy as opposed to improving technique or stimulation process' in effort to improve the general usefulness of massage. This study provides a potential breakthrough in grounding approach by combining simultaneously and precisely targeted grounding with the mechanical stimulation offered by massage.

Grounding massage is the controlled movement of bioelectric charge between the Earth and the subject to enable recovery from an unstable charge to a stable charge. With the aid of a grounding massager, an operator may charge or discharge the bioelectric system.

Different studies clearly show the physiology of massage as described above. Massage needs more mechanistic research to understand its immunomodulatory benefits better. Researchers should concentrate on the temporal structure of different inflammatory cell populations while minimizing the disturbing impact of multiple massage sessions combined with simultaneous earthing.

Since different cell-signaling pathways should be triggered with varying massage techniques, special attention should be paid to the methods combined. Establishing a favorable inflammatory environment facilitates repair and identifies the signaling pathways that grounding massage effects can provide.

Grounding massage needs to be included in the clinical practice of preventive, complementary, and lifestyle medicine, as it can possibly improve these approaches' effectiveness.

\section{CONCLUSION}

Grounding simultaneously combined and geographically targeted with mechanical massage is a non-invasive and nonpharmacological intervention with multidimensional functions and advantages. New findings substantiate the conventional arguments and confirm that grounding, when simultaneously combined and geographically targeted with mechanical massage, may be beneficial in treating various acute and chronic diseases. Grounding massagers can deliver generous benefits in pain relief and positive outcomes without significant adverse effects. Continued research in this area is necessary and justified.

\section{REFERENCES}

[1] Astin JA. Why patients use alternative medicine: results of a national study. JAMA. 1998 May 20;279(19):1548-53. DOI: 10.1001/jama.279.19.1548. PMID: 9605899.

[2] Barnes PM, Powell-Griner E, McFann K, Nahin RL. Complementary and alternative medicine use among adults: The United States, 2002. Adv Data. 2004;343:1-19.

[3] Best TM, Hunter R, Wilcox A, Haq F. Effectiveness of sports massage for recovery of skeletal muscle from strenuous exercise. Clin J Sports Med. 2008;18(5):446-460. [PubMed] [Google Scholar].

[4] Gross, A., Kay, T. M., Paquin, J. P., Blanchette, S., Lalonde, P., Christie, T., \& Cervical Overview Group. (2015). Exercises for mechanical neck disorders. Cochrane Database of Systematic Reviews, (1).

[5] Brosseau, L., Wells, G. A., Poitras, S., Tugwell, P., Casimiro, L., Novikov, M., \& Cohoon, C. (2012). Ottawa Panel evidence-based clinical practise guidelines on therapeutic massage for low back pain. Journal of bodywork and movement therapies, 16(4), 424-455.

[6] Dryden, T., Baskwill, A., \& Preyde, M. (2004). Massage therapy for the orthopaedic patient: a review. Orthopaedic Nursing, 23(5), 327 332 .

[7] Weerapong, P., Hume, P. A., \& Kolt, G. S. (2005). The mechanisms of massage and effects on performance, muscle recovery and injury prevention. Sports medicine, 35(3), 235-256.

[8] Field, T. M. (1998). Massage therapy effects. American Psychologist, 53(12), 1270. 
[9] Sagar, S. M., Dryden, T., \& Wong, R. K. (2007). Massage therapy for cancer patients: a reciprocal relationship between body and mind. Current Oncology, 14(2), 45.

[10] Moyer, C. A., Rounds, J., \& Hannum, J. W. (2004). A meta-analysis of massage therapy research. Psychological Bulletin, 130(1), 3 .

[11] Ober C, Sinatra ST, Zucker M. Earthing: the most important health discovery ever? Basic Health Publications, Inc.; 2010.

[12] Chevalier G. The effect of grounding the human body on mood. Psychological Reports. 2015 Apr;116(2):534-42

[13] Oschman, J. L., Chevalier, G., \& Brown, R. (2015). The effects of grounding (earthing) on inflammation, the immune response, wound healing, and prevention and treatment of chronic inflammatory and autoimmune diseases- Journal of Inflammation Research, 8, 83.

[14] Sokal, K., \& Sokal, P. (2011). Earthing the human body influences physiologic processes. The Journal of Alternative and Complementary Medicine, 17(4), 301-308.

[15] Guyton AC. Nervous regulation of the circulation and rapid control of arterial pressure. Textbook of medical physiology. 1991:194-204.

[16] Wang K, Liu J. Needling sensation receptor of an acupoint supplied by the median nerve-studies of their electrophysiological characteristics. The American journal of Chinese medicine. 1989;17(03n04):145-55.

[17] Becker, Robert O. "Exploring new horizons in electromedicine." (2004): 17-18

[18] Oschman, J. L., Chevalier, G., \& Ober, A. C. (2015). 38 Biophysics of Earthing (Grounding) the Human Body.

[19] Amalu, W. (2004) Medical thermography case studies. Unpublished study.

https://www.earthinginstitute.net/wpcontent/uploads/2019/02/thermog raphycasehistories2004.pdf.

[20] Chevalier G, Sinatra ST, Oschman JL, Delany RM. Earthing (grounding) the human body reduces blood viscosity—a major factor in cardiovascular disease. The journal of alternative and complementary medicine. $2013 \mathrm{Feb}$ 1;19(2):102-10.

[21] Chevalier, G. (2014). Grounding the human body improves facial blood flow regulation: results of a randomized, placebo-controlled pilot study-Journal of Cosmetics, Dermatological Sciences and Applications, 4(05), 293.

[22] Ghaly, M., \& Teplitz, D. (2004). The biologic effects of grounding the human body during sleep as measured by cortisol levels and subjective reporting of sleep, pain, and stress. Journal of Alternative \& Complementary Medicine, 10(5), 767-776.

[23] Elkin, H. K., \& Winter, A. (2018). Grounding Patients with Hypertension Improves Blood Pressure: A Case History Series Study. Altern Ther Health Med, 24(6), 46-50.

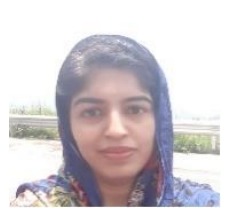

Madiha Khalid was born 11.9.1985 in Lahore.

M. Phil clinical Pharmacy, Humdard University, Lahore.

She works as a lecturer in the University of Lahore for about six years in the Pharmacy Department.

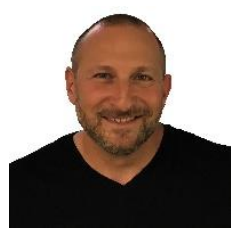

Jeremy Madvin was born 11.22.1967 in Los Angeles, USA.

MBA International Business, University of Redlands, United States. 